\title{
Effect of normal and atheromatous aortic tissue on platelet aggregation in vitro
}

\author{
C. R. M. PRENTICE, G. P. MCNiCOL, AND A. S. DOUGlas \\ From the University Department of Medicine, Glasgow Royal Infirmary
}

SYNOPSIS An investigation was made into the platelet-aggregating ability of human aortic tissue. It was found that potent aggregating substances are present in the vascular wall but that the degree and character of platelet aggregation produced was dependent on the method employed in the preparation of the tissue for study.

The supernatants of aortic tissue homogenates were found to produce potent irreversible platelet aggregation that occurred after a latent period of one to two minutes. The activity was heat labile, destroyed by collagenase, sedimented by ultracentrifugation, and was considered to be identical or similar to collagen. An extract obtained by boiling aortic tissue with saline was found to cause less powerful reversible platelet aggregation that occurred within 15 to 30 seconds' contact with platelets; the activity was not affected by heating, incubation with collagenase, or ultracentrifugation, and was considered to be due to adenosine diphosphate. Atheromatous aortic tissue when extracted by either method was found to contain less than one half of the potency of normal tissue in causing platelet aggregation.

Attention has recently been drawn to the role played by platelet aggregation in forming arterial thrombi ('Biological aspects of occlusive vascular disease', 1964). Reaction between the platelets and the injured or altered arterial wall is thought to occur as an early event during haemostasis in vivo, and may form the earliest stage of mural thrombosis as well as being a precursor of atheromatous arterial disease (Duguid, 1955). The present communication reports on the degree and character of platelet aggregation produced by the human arterial wall.

Bounameaux $(1959,1961)$ and Hughes $(1962)$ in animal studies noted that extracts of blood vessel were able to aggregate platelets, and the active principle was later characterized as collagen by Zucker and Borrelli (1962) using human material. Hovig (1963a and b) demonstrated that collagen could aggregate platelets by causing the liberation of adenosine diphosphate from the platelets themselves. Although Hellem (1960) was unable to show that the arterial wall increased the adhesiveness of platelets, Mitchell and Sharp (1964) obtained an arterial extract by boiling rabbit aorta in saline which caused platelet aggregation in vitro.

These observations suggest that there are two separate factors within the arterial wall, one heat labile and the other heat stable, capable of causing platelet

Received for publication 24 February 1966. aggregation, and it was decided to investigate their qualities more fully, and in addition to make a comparison between the activity of normal and atheromatous vessel.

\section{MATERIALS AND METHODS}

Platelet aggregation was assessed by a modification of the method of Born and Cross (1963) measuring changes in the optical density of the plasma. Platelet-rich plasma, $2 \mathrm{ml}$, was pipetted into a transparent cuvette, placed in an Evans Electro Selenium Ltd. titrator connected to a type 20 unigalvo, and stirred with a magnetic stirrer. In this instrument a beam of light is passed through the plasma in the cuvette and the initial optical density is measured. When platelet aggregation occurs the optical density falls, the degree of fall corresponding to the extent of platelet aggregation in the plasma. The initial optical density as measured in arbitrary units on the galvanometer was set at 60 and readings were taken at 30 -second intervals following addition of the test substance. A difficulty was encountered using the concentrated solutions in that the extracts from atheromatous tissue were more turbid than those from normal tissue masking the fall in optical density due to platelet aggregation. However, by using dilute solutions, e.g. 1 in 256 , the effect of turbidity was greatly reduced and acceptable comparisons could be made. All experiments were carried out at room temperature.

PLATELET-RICH PLASMA Whole blood, taken from healthy subjects by clean venesection using plastic dis- 
posable syringes, was added to $1 / 10$ volume of $3.8 \mathrm{w} / \mathrm{v}$ sodium citrate solution in siliconized containers and centrifuged for five minutes at $600 \mathrm{~g}$ at $4^{\circ} \mathrm{C}$. Platelet-rich plasma was pipetted off and stored in ice until use. The experiments were started immediately after preparation of the platelet-rich plasma.

Aortic tissue was taken at necropsy from normal and atheromatous areas of vessel as described previously (Prentice, McNicol, and Douglas, 1965). Aortic extracts were prepared by two different methods.

Homogenate Portions of tissue, each of $2 \mathrm{~g}$., were chopped into small pieces about $5 \mathrm{~mm}$. square and homogenized in a Quickfit homogenizer at room temperature with $20 \mathrm{ml}$. of $0.85 \%$ saline for 15 to 30 minutes until a creamy granular suspension was obtained. This was centrifuged at $600 \mathrm{~g}$ for 10 minutes and the supernatant pipetted off and stored at $-20^{\circ} \mathrm{C}$ in $1 \mathrm{ml}$. aliquots. This extract will be referred to as 'aortic homogenate'.

Boiled extract Pieces of aorta, each of 2 g., were chopped up into pieces approximately $5 \mathrm{~mm}$. square and boiled with $12 \mathrm{ml}$. of $0.85 \%$ saline for 20 minutes. The supernatant liquid was poured into a centrifuge tube, made up to $12 \mathrm{ml}$., by the addition of a further $0.85 \%$ of saline, centrifuged at $600 \mathrm{~g}$ for five minutes, and the supernatant stored at $-20^{\circ} \mathrm{C}$. in $1 \mathrm{ml}$. aliquots. This preparation will be referred to as 'boiled extract' of aortic tissue.

Dialysis of aortic extracts was performed for 24 hours at $4^{\circ} \mathrm{C}$. against 100 times their volume of $0.85 \%$ saline. In an attempt to detect small amounts of activity in the dialysate the solution was concentrated by freeze drying and the residue resuspended in $2 \mathrm{ml} .0 \cdot 85 \%$ saline.

Lipid extraction from the aortic tissue was achieved by shaking with 2:1 chloroform:methanol. Following separation with $0.85 \%$ saline for 12 hours the solution was evaporated to dryness and the lipid residue resuspended in $1 \mathrm{ml}$. saline.

Adenosine diphosphate, adenosine, and collagenase were supplied by Sigma Chemical Co., St. Louis, Missouri, U.S.A.

\section{RESULTS}

Figure 1 shows the effect of adding different concentrations of the boiled extract of normal and atheromatous tissue to platelet-rich plasma in Born's apparatus. Platelet aggregation began within 10 to 30 seconds and reached a maximum in two to five minutes. Thereafter disaggregation took place to an extent dependent on the concentration of the extract; with extract diluted 1 in 4 platelets were almost completely dispersed whereas with the undiluted extract small clumps of platelets were still present at 12 and 30 minutes after its addition. The atheromatous tissue had considerably less activity than the normal tissue and at best only one half the fall in optical density was noted as compared with the normal tissue. It will also be seen in Fig. 1 that the onset of aggregation and subsequent dispersal of platelets is very similar to that obtained by the addi-

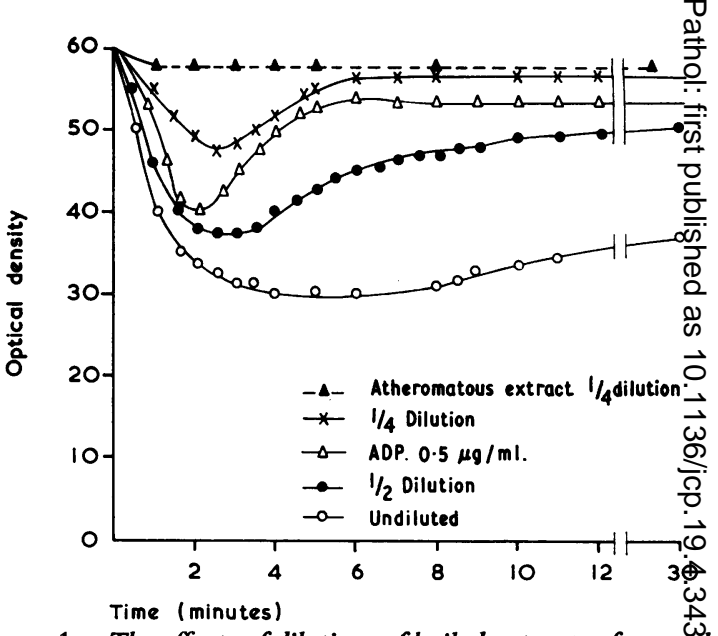

FIG. 1. The effects of dilutions of boiled extracts of normal aortic tissue in causing platelet aggregation.

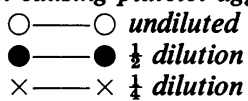

The effect of extract of atheromatous aortic tissue is at: shown.

$\Delta-\mathbf{\Delta} \frac{1}{4}$ dilution

The aggregation induced by a reference solution of adenosine diphosphate (A.D.P.) $0.5 \mu \mathrm{g} . / \mathrm{ml}$. is also illustrated. $\triangle \longrightarrow \triangle$

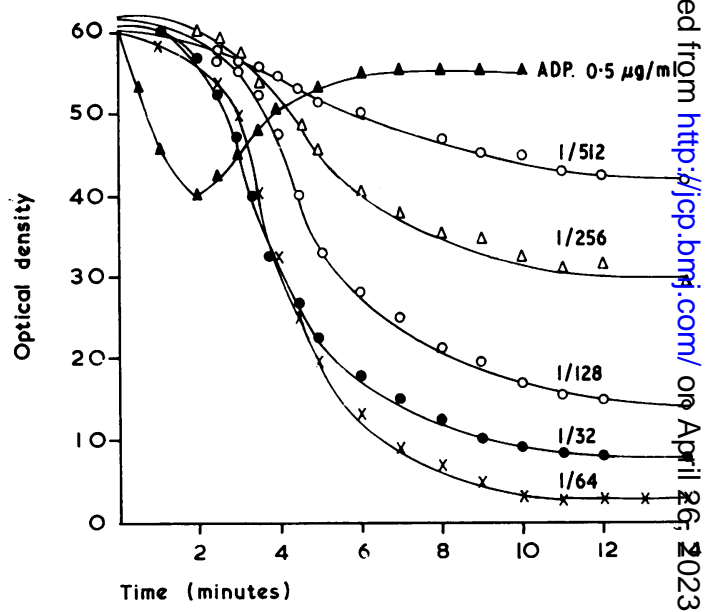

FIG. 2. The effect of addition of varying dilutions of homgenate of normal aortic tissue to platelet rich plasma. Eor comparison the effect of the reference solution of A.D.P. Fis also shown.

tion of adenosine diphosphate in a concentration 返 $0.5 \mu \mathrm{g}$. per millilitre of plasma.

In Fig. 2 the effect of adding different concentrations of the homogenate of normal aortic tissue 40 platelet-rich plasma is shown. The timing of platelet 
aggregation was entirely different from that produced by the boiled extract of aorta or by adenosine diphosphate. No aggregation occurred for about two minutes and thereafter there was a rapid fall in optical density to a minimum at about 15 minutes. The platelet aggregation induced was not reversible. Appreciable aggregating activity was still present at a dilution of 1 in 512; the extract at this dilution represented only $45 \mu \mathrm{g}$. of the original wet weight of the aortic tissue per millilitre of plasma. In this experiment also the potency of the atheromatous homogenate was less than half that of the normal tissue. The curve obtained by the addition of $0.5 \mu \mathrm{g}$. of adenosine diphosphate is also shown.

The effect of ultracentrifugation upon the two types of tissue is shown in Figure 3. The extracts

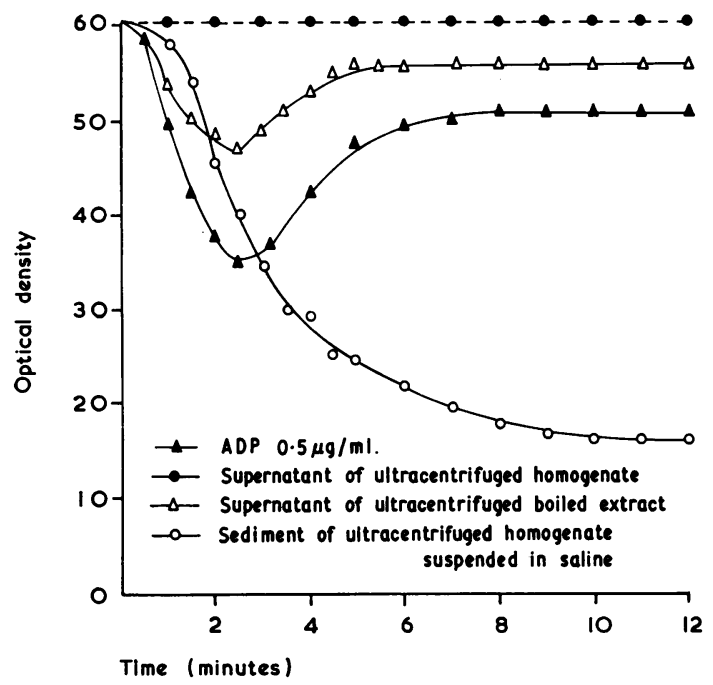

FIG. 3. The effect of ultracentrifugation upon the homogenate and boiled extract of normal aortic tissue.

were spun at 30,000 r.p.m. in a Beckman model $L$ preparative ultracentrifuge for 10 minutes. The supernatants were pipetted off and tested by the addition to platelet-rich plasma in Born's apparatus. The supernatant of the homogenate showed no platelet-aggregating activity. When the sediment was suspended in $0.85 \%$ saline to the same concentration as the original solution and added to platelet-rich plasma, platelet aggregation was again produced, the activity of the resuspended material being the same as that of the original extract.

The activity of the boiled extract was not destroyed or affected by ultracentrifugation. Similarly the activity of a control solution of adenosine diphosphate was unaffected by ultracentrifugation.
The effect of adenosine was tested by adding it in a concentration of $50 \mu \mathrm{g}$. per millilitre of plasma to the platelet-rich plasma 15 seconds before the addition of the aortic extract. Adenosine abolished the activity of both the aortic homogenate and boiled extract as well as that of the control solution of adenosine diphosphate.

Heparin added to platelet-rich plasma in concentrations varying from 5 to 50 i.u. per millilitre plasma and incubated at $37^{\circ} \mathrm{C}$. for 10 minutes before the addition of either type of extract had no effect upon their activity. Similarly heparin did not inhibit the activity of adenosine diphosphate.

The effect of temperature upon the homogenate of normal aorta is shown in Figure 4. Incubation at $60^{\circ} \mathrm{C}$. or $70^{\circ} \mathrm{C}$. for 10 minutes caused an approximate halving of activity. At $80^{\circ} \mathrm{C}$. only one quarter of the original activity was detected after 10 minutes. The effect of boiling the homogenate at $p \mathrm{H} 6.9$ was variable. In most cases slight residual activity was present after 10 minutes although in two of the six extracts tested activity was completely destroyed.

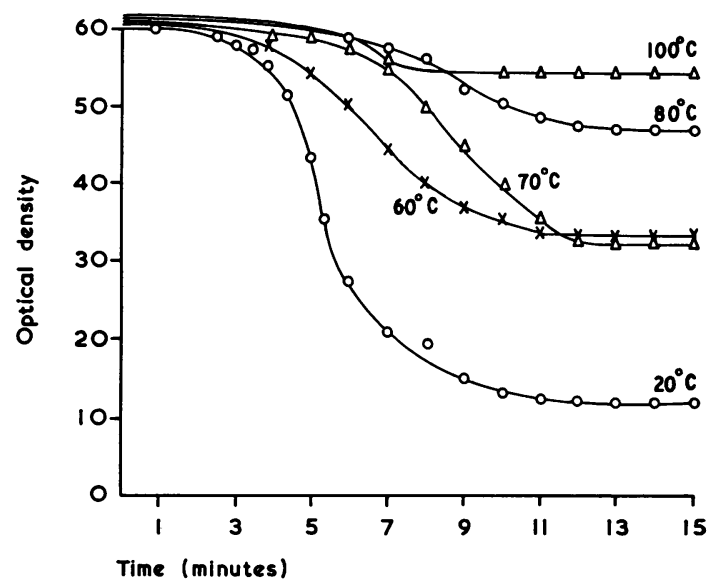

FIG. 4. The effect of heating the aortic homogenate to different temperatures for 10 minutes.

After 20 minutes at $100^{\circ} \mathrm{C}$. activity was destroyed in all the six extracts tested.

The boiled extract of aorta could be heated at $100^{\circ} \mathrm{C}$. for 10 minutes without significant loss of activity. A solution of adenosine diphosphate could also be boiled for 10 minutes without affecting its platelet-aggregating properties.

Storage of the homogenate in dilute solutions at $-20^{\circ} \mathrm{C}$. for two months did not affect its plateletaggregating abilities, but when the boiled extract was kept at $-20^{\circ} \mathrm{C}$. for two months it had become totally inactive. A dilute solution of adenosine diphosphate, 


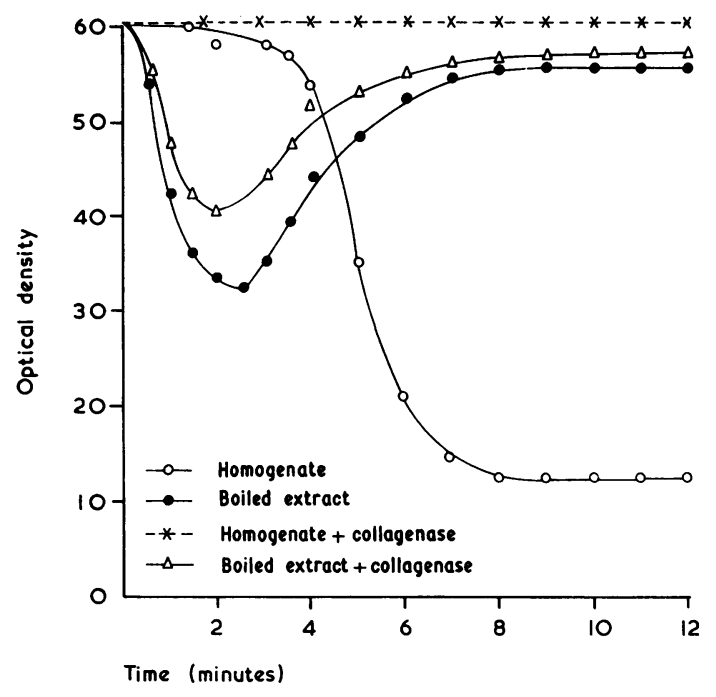

FIG. 5. The effect of collagenase upon the homogenate and boiled extract of normal aorta.

$10 \mu \mathrm{g}$. per ml., stored for the same period of time lost over half of its activity.

The effect of incubating the two types of tissue extract with collagenase is shown in Figure 5. Collagenase was prepared to give a final concentration of $1 \mathrm{mg}$. per millilitre plasma and incubated for one hour at $37^{\circ} \mathrm{C}$. with the aortic extracts which were then tested against platelet-rich plasma in Born's apparatus. Following incubation the activity in the homogenate was completely destroyed whereas that in the boiled extract was unaffected. Collagenase added directly to platelet-rich plasma in Born's apparatus was inactive in producing aggregation.

Lipid extraction was carried out on both normal and atheromatous pieces of aortic tissue. The chloroform-methanol-soluble material when evaporated and resuspended in saline had no effect when added to platelet-rich plasma.

\section{DISCUSSION}

It is apparent from these studies that human aortic tissue contains potent platelet-aggregating substances, the precise nature of the activity depending on the method of tissue extraction used. Homogenization of the tissue produces a potent heat-labile substance which causes powerful irreversible aggregation of platelets after a latent period of two to three minutes, whereas the solution obtained by boiling the tissue with $0.85 \%$ saline has less potent plateletaggregating activity which causes reversible platelet aggregation after contact for only 15 to 30 seconds.
It is very probable that the activity of the hom genized extract is due to collagen as it is specifica destroyed by collagenase as well as being no dialysable and sedimented by ultracentrifugation. The studies of Bounameaux (1959, 1961), Hughes (1962), Zucker and Borrelli (1962), and Hovig (196 作 and b) have shown that collagen found in severif types of connective tissue has potent platele aggregating ability. In contrast, the activity of the boiled extract is identical to that of adenosine diphosphate and is most likely to be due to adenosine diphosphate released from the aortic tissue during boiling. Like adenosine diphosphate it was noted be heat stable, unaffected by collagenase or ultra centrifugation; activity was gradually lost in dilute solutions at $-20^{\circ} \mathrm{C}$. Mitchell and Sharp (1964) have isolated a similar substance by boiling rabbit aorta although its identity as adenosine diphosphate wăgs not established. As Hellem (1960) was unable to detect such activity in human aorta, it is possible that the mechanism by which platelets are made adhesife is not identical to that causing aggregation. The adenosine diphosphate-like activity of the boil extract is not as potent as the collagen-like activity of the homogenate, but this may be due to incorn plete extraction of the adenosine diphosphate.

The platelet-aggregating activity of atheromatows aorta was less than half that of normal tissue wheू extracted by either method. As an atheromatows plaque contains relatively less connective tissue and more lipid than normal intima it is likely that both the collagen-like and the adenosine-diphosphate-like activity within aorta are produced by the connecti站 tissue rather than by lipid. The lipid extracted from atheromatous plaques had no platelet-aggregating activity but no definite conclusions can be draw from this as the studies were carried out in saline suspensions in which the lipids were insoluble Haslam (1964) and Kerr, Pirrie, MacAulay, and Bronte-Stewart (1965) have shown that solutions $+\frac{t}{m}$ vitro of fatty acids and phospholipids can cause platelet aggregation and it is clear that the physic $\tilde{\theta}$. chemical state in which the lipids are present is fundamental importance to the effect which they produce on platelet aggregation. The observation that the platelet-clumping activity of both aortic homogenate and boiled extract is abolished by adenosine suggests that adenosine diphosphate $\stackrel{\$}{\mathbb{1}}$ involved in the clumping initiated by both suBsstances. Hovig (1963a and b) has demonstrated that the clumping activity of collagen involves the liber8tion of adenosine diphosphate and that adenosiat inhibits the reaction between platelets and adenosige diphosphate. The failure in this experimental model of heparin in the concentrations tested to dimini迎 the aggregation of platelets by either type of extragt 
may have relevance to the therapeutic use of heparin in attempts to prevent in vivo the early stages of platelet aggregation.

It should be noted that aortic homogenate causes platelet aggregation only after a latent period of one to two minutes; the delay may be due to the reaction or sequence of reactions which takes place between collagen and the platelets. Further, as the collageninduced clumping is irreversible, it can be compared to the irreversible fusion or 'viscous metamorphosis' induced by thrombin; it is possible that collagen acts by a mechanism more complex than directly causing release of adenosine diphosphate from the platelets, as adenosine-diphosphate-induced clumping is reversible.

The potential ability of human arterial wall to cause platelet aggregation indicates that in health the endothelium of the vessel must be of importance in protecting the platelets from exposure to subendothelial tissues and that in areas of endothelial damage it is likely that platelet aggregates will be formed. The finding that platelet clumps do not disperse spontaneously following exposure to tissue homogenates suggests that if once formed within the arterial circulation in vivo such aggregates could provide the nidus for a permanent mural thrombus.

We are grateful to Professor E. M. McGirr and Dr. E. G. Oastler for their interest in this work. Thanks are due to the Medical Research Council for financial support.

\section{REFERENCES}

Biological Aspects of Occlusive Vascular Disease (1964), edited by D. G. Chalmers and G. A. Gresham, Cambridge University Press, London.

Born, G. V. R., and Cross, M. J. (1963). J. Physiol. (Lond.), 168, 178. Bounameaux, Y. (1959). C.R. Soc. Biol. (Paris), 153, 865.

(1961). Thrombos. Diathes. haemorrh. (Stuttg.), 6, 504.

Duguid, J. B. (1955). Brit. med. Bull., 11, 36.

Haslam, R. J. (1964). Nature (Lond.), 202, 765.

Hellem, A. J. (1960). Scand. J. clin. Lab. Invest., 12, Suppl. 51.

Hovig, T. (1963a). Thrombos. Diathes. haemorrh. (Stuttg.), 9, 248. (1963b). Ibid., 9, 264.

Hughes, J. (1962). Ibid., 8, 241.

Kerr, J. W., Pirrie, R., MacAulay, I., and Bronte-Stewart, B. (1965). Lancet, 1, 1296.

Mitchell, J. R. A., and Sharp, A. A. (1964). Brit. J. Haemat., 10, 78. Prentice, C. R. M., McNicol, G. P., and Douglas, A. S. (1965). J. clin. Path., 19, 154.

Zucker, M. B., and Borrelli, J. (1962). Proc. Soc. exp. Biol. (N.Y.), 109, 779. 